\title{
ÊTRE ENSEIGNANTE D'ÉDUCATION PHYSIQUE DANS UN CONTEXTE NON RÉGLEMENTE DE MIXITÉ : ÉTUDE EXPLORATOIRE EN SUISSE (GENÈVE)
}

\section{Manon Cattani et Grégory Quin}

De Boeck Supérieur | «Staps»

2019/1 n 123 | pages 121 à 132

ISSN 0247-106X

ISBN 9782807393370

Article disponible en ligne à l'adresse :

https://www.cairn.info/revue-staps-2019-1-page-121.htm

Distribution électronique Cairn.info pour De Boeck Supérieur.

(C) De Boeck Supérieur. Tous droits réservés pour tous pays.

La reproduction ou représentation de cet article, notamment par photocopie, n'est autorisée que dans les limites des conditions générales d'utilisation du site ou, le cas échéant, des conditions générales de la licence souscrite par votre établissement. Toute autre reproduction ou représentation, en tout ou partie, sous quelque forme et de quelque manière que ce soit, est interdite sauf accord préalable et écrit de l'éditeur, en dehors des cas prévus par la législation en vigueur en France. Il est précisé que son stockage dans une base de données est également interdit. 


\title{
Être enseignante d'éducation physique dans un contexte non réglementé de mixité : étude exploratoire en Suisse (Genève) \\ Teaching physical education in an unregulated co-educational context: An exploratory study in Switzerland (Geneva)
}

\author{
Manon CATTANI \\ Institut des Sciences du Sport \\ Université de Lausanne \\ manon.cattani@etu.unige.ch \\ Grégory QUIN \\ Maître d'enseignement et de recherche \\ Institut des Sciences du Sport \\ Université de Lausanne \\ gregory.quin@unil.ch
}

Résumé : Rendue obligatoire tardivement, en 1972, l'éducation physique pour les filles dans les systèmes scolaires de Suisse est désormais un acquis et une réalité sur l'ensemble du territoire national et pour toute la durée de la scolarité obligatoire. Pourtant, les systèmes scolaires helvétiques n'ont jamais entamé de réflexion sur les conditions de cette entrée des filles dans une branche singulière de l'École. Dans ce cadre, notre ambition est de proposer une analyse de cette situation, en cherchant à comprendre les logiques de l'engagement des femmes dans l'enseigrnement de l'éducation physique et les mutations d'une branche scolaire, dont l'une des spécificités est de véhiculer des représentations de genre très puissantes et encore très effectives. Dans ce cadre, nous avons entrepris de réaliser des entretiens avec des enseignantes de différentes générations depuis les années 1970 pour chercher à circonscrire les potentielles transformations de l'engagement des femmes enseignantes d'éducation physique pour le canton de Genève. À la croisée d'une histoire de l'éducation physique et d'une sociologie d'une profession singulière, nos conclusions soulignent à la fois la permanence de certaines représentations " genrées ", les lentes transformations d'un métier et les difficultés inhérentes au maintien d'une non-régulation de la situation de mixité en éducation physique et plus largement en contexte scolaire.

Mots cLés : éducation physique, femmes enseignantes, Suisse, genre

L'éducation physique devient une branche obligatoire dans les écoles helvétiques suite à une réforme de l'organisation militaire du pays en 1874, selon une chronologie relativement semblable aux autres pays européens (Burgener, 1984 ; Bussard, 2007 ; Cordoba \& Lenzen, 2018). Comme ailleurs, c'est alors une gymnastique d'inspiration militaire et exclusivement masculine, dont les enseignants sont d'anciens instructeurs militaires, et les filles ne reçoivent de l'éducation physique que dans certaines écoles, principalement en ville et de manière non coordonnée (Bussard, 2007 ; Fontaine, 2015). L'éducation physique s'impose dès lors comme une branche scolaire fédérale, dans un système politique où ce sont les cantons qui assurent la gouvernance des systèmes scolaires. Dans les faits, il faut attendre la publication d'un nouveau manuel fédéral entre 1957 et 1962 pour voir apparaitre des contenus spécifiques pour les filles (Bussard, 2001). Il s'agit donc de la première tentative de développement d'une éducation physique plus ouverte à l'ensemble des élèves, même si, 
depuis les années 1930 et 1940, des femmes obtiennent la nouvelle certification fédérale de maitresse d'éducation physique (Quin, 2016). Ainsi, à Lausanne, dès les années 1940 , on compte environ une femme pour deux hommes dans les sessions de formation universitaire (Quin, 2011). Pour autant, dans les années 1960, l'éducation physique des filles n'est pas encore une obligation légale. C'est uniquement au moment du vote de la première loi fédérale sur la gymnastique et le sport en 1972, que le législateur élargit l'obligation de l'éducation physique scolaire aux jeunes filles en âge de scolarité obligatoire - de 6 à 16 ans (Burgener, 1984 ; Quin, 2015) ; soit une année seulement après l'obtention par les femmes du droit de vote au niveau fédéral (Studer, 1996).

Néanmoins, malgré une dynamique sociale forte, les systèmes scolaires helvétiques n'ont jamais réellement entamé de réflexion sur les conditions de cette entrée des filles dans une branche singulière de l'École, pas plus avant 1972 que lors des dernières décennies. En effet, à la différence de la France, les institutions scolaires suisses n'imposent pas de cadre légal homogène sur la mixité, rendant de fait la réalité très contrastée dans les différents cantons et parfois au sein même d'un établissement, à la fois pour les élèves qui ne sont pas traité·e·s sur un pied d'égalité et pour les enseignant·e.s qui sont chargéee-s de la branche. Aucune prescription n'est établie par les autorités fédérales - Commission fédérale de Gymnastique et de Sport ou Conférence intercantonale des Directeurs de l'Instruction publique -, et finalement, chaque canton est laissé libre pour la mise en œuvre de la nouvelle obligation et des conditions de mixité que cela engendre. Dès lors, il n'est pas surprenant que ces différents systèmes scolaires se retrouvent influencés par des dynamiques internationales de remise en cause de la mixité (Bréau \& Lentillon-Kaestner, 2017). À Genève, il existe ainsi des établissements dans lesquels les enseignements d'éducation physique sont non mixtes, dans d'autres établissements cela dépend des niveaux d'enseignement ou des activités physiques sportives et artistiques enseignées et surtout, certain·e·s enseignant·e.s n'enseignent soit qu'à des garçons, soit qu'à des filles.

Jusqu'à présent, à la différence de pays voisins comme la France, ce dont attestent les travaux récents de Vanessa Lentillon, de Loïc Szerdahelyi ou encore de Jacques Gleyse (Lentillon, 2009 ; Szerdahelyi, 2009 ; Gleyse, 2010), en Suisse les recherches sur ces questions restent très rares, et finalement les historien.ne's ou les sociologues ne se sont pas véritablement emparé·e·s de ces thématiques pour questionner les transformations de l'enseignement de l'éducation physique au cours des dernières décennies. Tout au plus, nous pouvons citer les travaux de Véronique Czaka (2012), mais ils sont avant tout focalisés sur la période antérieure à la Première Guerre mondiale, ou ceux plus contemporains réalisés autour de l'équipe de Vanessa Lentillon (Lentillon-Kaestner et al., 2018). Au final, la période consécutive à la Seconde Guerre mondiale reste très peu étudiée, malgré les potentialités explicatives de cette branche scolaire pour la compréhension du fonctionnement de la société suisse dans son ensemble. Récemment, dans le cadre du travail de doctorat d'Antoine Bréau, des analyses plus poussées sur les situations de mixité et de non-mixité en éducation physique ouvrent des perspectives nouvelles, notamment sur les avantages et les désavantages de chaque situation. Ainsi, pour ces chercheur.e.s, l'enjeu des travaux à venir est "d'identifier les manières de "faire le genre" (doing gender) et de repérer des configurations typiques d'actions et d'intentions au sein desquelles le genre s'exprime et participe à la construction de l'identité de chacun et chacune » (Bréau, Lentillon-Kaestner \& Hauw, 2016, p. 125).

Dès lors, à l'aune des manques encore existants dans la littérature et des conclusions de ces travaux, l'ambition de notre contribution est multiple. À partir de l'exemple genevois, nous avons souhaité comprendre les logiques de l'engagement des femmes dans l'enseignement de l'éducation physique, les mutations 
d'une branche scolaire (Moreau, 2011) à la gouvernance singulière, dont l'une des spécificités est de véhiculer des représentations de genre très puissantes et les situations de mixité - ou non - constatées dans l'enseignement secondaire. Être une femme enseignante d'éducation physique continue d'être parfois perçu comme un métier complexe, pour lequel les femmes auraient "quelque chose à prouver ", à la fois vis-à-vis de leurs collègues et des différent.e.s acteurs/actrices des systèmes scolaires, mais aussi des élèves, dont les représentations sont loin d'être devenues plus «neutres " ces dernières décennies (Cogérino, 2007).

Dans ce cadre, nous avons entrepris de réaliser des entretiens "récits de vie " avec des enseignantes de différentes générations ${ }^{1}$, entrées dans la profession depuis les années 1970, pour chercher à circonscrire les transformations de l'engagement et du vécu des femmes enseignantes d'éducation physique pour le canton de Genève (Cattani, 2017). À travers ces entretiens et l'étude d'un cas cantonal singulier, notre ambition est d'appréhender la féminisation de la profession - relativement stable en proportion depuis les années 1970 et plus largement les recompositions des frontières de genre autour de l'éducation physique scolaire et du sport (Mennesson, 2005).

À la croisée d'une histoire de l'éducation physique (Cordoba \& Lenzen, 2018) et d'une sociologie d'une profession singulière (Maruani, 1998 ; Essen \& Rogers, 2003 ; Cacouault-Bitaud, 2007), nous envisageons de poser quelques premiers jalons pour appréhender la permanence de certaines représentations de genre, les transformations d'un métier et les difficultés inhérentes à une situation non réglementée de mixité en éducation physique et plus largement en contexte scolaire. Pour présenter nos résultats, nous nous attacherons à suivre le parcours des enseignantes depuis leur formation jusqu'à leur situation professionnelle actuelle, en abordant leur ressenti face aux élèves garçons et filles et après avoir exposé leurs interactions avec leurs collègues et leurs directions ${ }^{2}$, au sein de leurs établissements.

\section{De la formation au métier D'ENSEIGNANTE D'EP}

Dans le cadre de notre recherche, les enseignantes d'éducation physique interrogées n'ont pas déclaré avoir choisi cette carrière pour aller au-delà des normes socialement établies et du caractère " masculin » de leur discipline. En effet, elles évoquent plus aisément un intérêt précoce pour la pratique sportive ou pour le domaine de l'enseignement, influencé par des modèles incarnés par d'autres enseignantes ou par le contexte familial. De plus, leurs motivations sont davantage pédagogiques qu'en lien avec les conditions de travail.

Cependant, leur choix de devenir enseignante d'éducation physique a pu parfois engendrer une réactualisation de stéréotypes de genre dans l'entourage, au moment de la prise de décision. C'est le cas, par exemple, d'Hanna qui explique qu'on a pu remettre en doute sa capacité à tenir une classe "vu qu'eelle est] une femme, qu'[elle n'est] pas très grande, qu'[elle n'a] pas la voix qui porte» (24 février 2017, entrée en fonction en 2014), et alors que son expérience n'est pas encore très longue.

Durant la formation, les enseignantes interrogées ne constatent pas de réel déséquilibre pour ce qui est du nombre de femmes et d'hommes, comme le confirment les statistiques des différentes volées de la formation genevoise, entre 1970 et $2016^{3}$. Si ces données sont avant tout quantitatives, il est possible de souligner que le ratio entre les femmes et les hommes - environ une femme pour deux

\footnotetext{
1 Trois générations composent notre corpus : les noms d'emprunt commençant par A, B ou C ont commencé dans les années 1970 et 1980, les noms en D, E et F au début des années 2000 et les noms en G et H depuis le milieu des années 2010.

2 Dans les systèmes scolaires suisses ce sont les directions d'établissements qui ont la charge du recrutement des équipes pédagogiques.

3 Ces données ont été construites sur la base d'une lecture des archives de l'Institut des Sciences du Mouvement et de la Médecine du Sport de l'Université de Genève.
} 
hommes - demeure finalement assez stable au fur et à mesure de l'universitarisation de la formation en Suisse romande (Hofstetter, Schneuwly \& Lussi Borer, 2009 ; Quin, 2016).

De la même manière, ce ratio semble perdurer dans l'exercice du métier à l'école secondaire, puisque nos recherches dans les statistiques genevoises indiquent les chiffres suivants : au secondaire I, 41 femmes et 97 hommes enseignent l'éducation physique en 2017, pour 45 femmes et 95 hommes au secondaire $\mathrm{II}^{4}$. Nous constatons ainsi que les femmes enseignantes d'éducation physique ne sont pas plus nombreuses par rapport aux hommes au cycle d'orientation - secondaire I. Il n'existe donc pas véritablement de corrélation avec le nombre moins important de groupes mixtes d'élèves au secondaire I comparé au secondaire II, comme cela sera détaillé par la suite. De plus, cela semble battre en brèche l'idée que les femmes seraient sous-représentées dans les segments les plus prestigieux de l'enseignement secondaire - en l'occurrence le secondaire II - (Moreau, 2011). Ces constats liminaires nous invitent évidemment à entrer dans une compréhension plus précise des logiques de genre qui entourent l'enseignement de l'éducation physique.

À la question : " enseigner l'éducation physique, est-ce un métier d'homme ou de femme?", les enseignantes, quelle que soit leur ancienneté, répondent en grande majorité qu'il s'agit d'une profession "mixte». Toutefois, elles évoquent ensuite des différences avec leurs collègues masculins d'ordre psychologique et en lien avec la personnalité pour parfois justifier leur légitimité à exercer ce métier ainsi que leur place dans la profession, participant ainsi elle-même parfois à une reproduction "naturalisante " des différences hommes/femmes sous couvert d'une mobilisation de " traits de personnalité " supposément féminins. De plus, certaines d'entre elles différencient l'enseignement tout court, domaine davantage investi par les femmes, du sport en tant que tel, lui-même étant encore perçu comme très masculin. C'est le cas par exemple d'Eva, lorsqu'elle avance que l'enseignement « est vachement plus pour les femmes. [...] On transmet, on aime donner, on aime partager, [on a] plus de sensibilité qu'un homme [...], je pourrais avoir l'impression que c'est plus fait pour les femmes " (9 février 2017, entrée en fonction en 2001). En revanche, à ce stade, aucune d'entre elles n'aborde les différences entre les convictions éducatives ou « la façon d'enseigner » (Cogérino \& Joux, 2005). Toutefois, la majorité des femmes interrogées dans le cadre de cette recherche mettent en avant leur "sensibilité " comme force pour la profession. Hanna fait part d'un sentiment de fierté quant au fait d'être une femme enseignante d'éducation physique et affirme sa volonté "de bousculer un peu les choses". Elle ajoute qu'elle « trouve que les profs de sport femmes, cela contribue à l'égalité vu que c'est quand même un peu à la base un métier masculin " (24 février 2017, entrée en fonction en 2014). Ainsi, certaines enseignantes s'appuient sur des stéréotypes de genre dans leurs commentaires, tout en démontrant une volonté de s'en détacher, mettant en lumière les contradictions face auxquelles elles se trouvent. De la même manière, les exigences physiques du métier sont aussi parfois abordées sous un jour "stéréotypé ", comme pour Daniela, qui explique qu'« il faut être en forme, parce que c'est costaud quand même. Ce n'est pas rien d'enseigner le sport » (10 février 2017, entrée en fonction en 2000).

\section{ENSEigner l'EP AU SEIN D'UN ÉTABLISSEMENT GENEVOIS}

De manière a priori paradoxale, la majorité des enseignantes interrogées nous ont affirmé que le fait d'être une femme leur a ouvert les portes de la profession. La raison principale

4 Dans les systèmes scolaires suisses, le « secondaire I » correspond à la scolarité secondaire obligatoire pour l'ensemble des classes d'âge - de 12 à 15 ans -, alors que le «secondaire II » correspond à une scolarité secondaire supérieure où les élèves ont été orienté·e·s vers différents débouchés universitaires ou professionnels. 
à cela est liée à la présence d'un nombre plus important de classes non mixtes dans les établissements genevois - particulièrement au secondaire I et par rapport aux cantons limitrophes - et à un présupposé des directions sur la nécessité d'avoir des femmes pour enseigner aux filles.

D’après les chiffres exposés précédemment, le ratio hommes/femmes est identique dans les différents niveaux de la scolarité secondaire genevoise. Ces résultats peuvent étonner, dans la mesure où une différence pourrait être explicable du fait que la mixité en éducation physique est davantage mise en place dans les établissements du secondaire II $^{5}$. Ainsi, les directions d'établissements du secondaire I engageraient des femmes pour enseigner aux classes de filles, ce qui aurait pu expliquer leur nombre plus conséquent au cycle d'orientation. À l'appui de ce constat, nous pouvons indiquer que Françoise est la seule femme - sur dix enseignants d'éducation physique - dans son établissement dans lequel la mixité est de mise.

Ainsi, les femmes interrogées évoquent les besoins en enseignantes pour assurer les cours d'éducation physique aux filles, comme l'explique Daniela : "Ils nous disaient quills cherchaient des femmes. C'est plus facile pour une femme de trouver une place en sport, pour enseigner, que pour un homme» (10 février 2017, entrée en fonction en 2000). Les enseignantes développent les mêmes structures argumentatives en évoquant la recherche de spécialistes pour assurer les activités « féminines », comme l'évoque Agnès : "Ils cherchaient quelqu'un qui enseignait la danse " (14 mars 2017, entrée en fonction en 1975). En effet, les directions, en plus d'une volonté d'équilibre entre les sexes au sein du groupe enseignant, cherchent fréquemment à engager des femmes enseignantes d'éducation physique pour enseigner aux classes de filles et ce, «depuis toujours» comme le relatent les plus anciennes. Ainsi, il n'est pas rare à Genève que les filles n'aient jamais eu affaire à un enseignant d'éducation physique homme, durant leur curriculum scolaire. De même, certaines enseignantes n'ont jamais enseigné à des garçons, mis à part dans certaines rares options mixtes.

$\mathrm{Au}$ sein des équipes enseignantes, les choses sont également singulières. En ce qui concerne leur relation avec la direction, qui pour la majorité des enseignantes ne pose pas de problème, deux d'entre elles ont fait part de comportements stéréotypés masculins ayant sans doute permis l'obtention du poste pour la première, et provoquant des tensions avec la direction pour la seconde. En effet, Gaïa suggère que sa personnalité « impressionnante [...], rentre-dedans [qui] ne ferme pas sa bouche, [...] qui en a sous le pied " (3 janvier 2017, entrée en fonction en 2012), aurait joué en sa faveur pour son engagement, comme s'il était attendu, pour le poste, d'avoir ce type de caractère. De son côté, Céline estime que le fait "d'être aussi carrée qu'un homme" (2 février 2017, entrée en fonction en 1985) a pu surprendre sa direction. Ainsi, ces deux femmes font usage de stéréotypes ayant trait à des caractéristiques plutôt positives - «en avoir sous le pied » et «ne pas se laisser marcher sur les pieds»-, qualifiées indirectement pour la première et directement pour la seconde comme étant des attitudes plutôt masculines et susceptibles de les avoir aidées dans leurs trajectoires professionnelles.

D'après les entretiens réalisés, la collaboration entre collègues d'éducation physique semble fonctionner dans les établissements dans lesquels travaillent ces femmes. Tout au plus, il apparait que la collaboration est plus aisée entre les enseignantes femmes, mais pour des raisons pratiques - partage des vestiaires, classes de filles... -, inhérentes à la structure non mixte de l'enseignement de l'éducation physique dans de nombreux établissements. Au niveau de la collaboration et d'après l'analyse des témoignages des enseignantes, nous

5 La majorité des établissements genevois du secondaire II propose un enseignement de l'éducation physique sous forme de système à options. Les élèves sont ainsi répartsi·e·s par groupes, d'après leurs choix pour les activités physiques sportives et artistiques proposées. 
pouvons penser que la "génération » et le type de formation suivie produit une ségrégation plus importante que celle du " genre » ou du « sexe ». C'est par exemple le cas de Céline qui raconte " avoir dî prouver quelque chose " (2 février 2017, entrée en fonction en 1985), c'est-à-dire qu'elle méritait sa place et qu'elle était compétente, à son arrivée dans l'un des premiers établissements dans lequel elle a travaillé. Ainsi, l'âge et l'expérience semblent être des facteurs aussi importants que le genre au regard des facilités de collaboration et d'intégration dans une équipe, notamment pour les conceptions pédagogiques différentes qui peuvent diviser les enseignants.

\section{3. ĖTRe ENSEIGNANTE D'EP FACE AUX ÉLÈVES DU SECONDAIRE}

La mixité au sein des établissements secondaires genevois n'étant pas normée par les autorités, chaque établissement a le choix de l'imposer ou non. Nous pouvons alors émettre l'hypothèse que le système - mixte ou non mixte - mis en place dans les établissements dépend d'un choix de direction, de contraintes organisationnelles - dans la préparation des horaires, par exemple - ou d'une demande de la part des enseignant.e.s. Sachant effectivement qu'il serait plus délicat d'imposer des classes mixtes à des enseignant.e.s n'ayant jamais enseigné dans ce type de regroupement et/ou ne souhaitant pas le faire que l'inverse - soit d'imposer des classes non mixtes à des enseignant·e.s en faveur de la mixité - ; nous pouvons avancer que les convictions des enseignant.e.s en faveur des groupes non mixtes en éducation physiques primeraient sur la mise en place généralisée de la mixité dans cette discipline.

Dans ce cadre, le point de vue des enseignantes au sujet de la mixité a été questionné et une réflexion sur la nature de leurs interactions avec les élèves filles et avec les élèves garçons a été menée dans le cadre des entretiens. Force est de constater que les avis des enseignantes d'éducation physique interrogées sur la question de la mixité au sein des leçons d'éducation physique sont partagés. En effet, chaque enseignante justifie son point de vue en évoquant des points positifs et négatifs et traite d'elle-même des différences de comportement entre les filles et les garçons durant les leçons d'éducation physique.

Pour motiver leur préférence pour la mixité ou la séparation des filles et des garçons, certaines enseignantes, comme Daniela, évoquent "les transformations physiologiques des filles et des garçons à l'adolescence " (10 février 2017, entrée en fonction en 2000) qui justifieraient une séparation des élèves par sexe, pour l'enseignement de l'éducation physique. D'autres mettent en avant l'aspect relationnel pour justifier leur préférence. C'est le cas de Béatrice qui affirme : "[aimer] mieux tirer les filles que devoir ralentir les garçons", avec lesquels elle explique "ne pas [être] à l'aise " (7 février 2017, entrée en fonction en 1985). Un autre argument évoqué est celui des interactions entre les élèves des deux sexes qui péjoreraient l'ambiance de travail ou provoquerait un "effacement " des filles en présence des garçons (DurandDelvigne \& Duru-Bellat, 1998), comme le confirme Céline : "Ces garçons qui se prennent trop pour des caïds et qui intimident les filles " (2 février 2017, entrée en fonction en 1985). Ainsi, la présence des garçons rendrait les filles dépendantes du système catégoriel de sexe et des stéréotypes le spécifiant (Mosconi \& Vouillot, 2013). Toutefois, l'argument inverse, soit de dire que la présence de l'autre sexe peut faire office de moteur, a également été avancé par Hanna : "Les filles ont envie de prouver leur place au sein de l'activité, les garçons, eux aussi, ne vont pas vouloir perdre la face » (24 février 2017, entrée en fonction en 2014). Enfin, une baisse du niveau de performance serait observable en contexte mixte et le niveau d'activité considéré comme faible dans les classes de filles.

Pour Gaiia, les préférences des enseignantes quant à la mise en place ou non de la mixité au sein des établissements sont liés à des raisons de sexe surtout, et de génération. Cela complète donc l'argument précédemment 
avancé, qui suggérait que les avis des enseignant.e.s quant à la mixité en éducation physique pouvaient compter pour le choix de la mise en place ou non de cette dernière dans établissements du secondaire à Genève. En effet, une certaine évolution du point de vue est repérable : les plus anciennes enseignantes ont pour point commun d'être opposées à la mixité au secondaire I voire également au secondaire II, et deux d'entre elles n'apprécient pas ou ne souhaiteraient pas enseigner aux garçons. De plus, elles ont toutes beaucoup, si ce n'est uniquement pour certaines, enseigné à des classes de filles. Il est probable que l'expérience de la mixité en tant qu'élève ou en tant qu'enseignante puisse jouer en la faveur d'une préférence pour la mixité et cela explique sans doute leur inclination pour la séparation des sexes. Plus les enseignantes sont jeunes, plus elles paraissent favorables ou intéressées par la mise en place de la mixité dans les leçons d'éducation physique. À noter que plusieurs de ces femmes enseignantes n'ont pas clairement pris position en faveur ou en défaveur de la mixité, en raison de leurs diverses expériences positives et négatives qui dépendaient, d'après elles, davantage du fonctionnement du groupe classe, qu'il soit mixte ou non.

Suite à la réflexion des enseignantes concernant leurs interactions avec leurs élèves, plusieurs d'entre elles ont pointé des différences entre les filles et les garçons, parfois de manière très stéréotypée. De plus, toutes admettent différencier leur enseignement et leur manière d'interagir selon qu'il s'agisse de filles ou de garçons, ne prenant ainsi pas en considération leurs élèves de manière similaire. Ainsi, Gaïa explique "utiliser un levier performance avec les garçons [et] un levier plutôt de santé chez les filles » (3 janvier 2017, entrée en fonction en 2012) pour construire les ressorts de la motivation. En effet, les femmes attribuent plus facilement un comportement de débutantes aux filles (Durand-Delvigne \& Duru-Bellat, 1998). Face aux garçons, que Béatrice qualifie de "plus compétitifs " (7 février 2017, entrée en fonction en 1985), Céline atténue son côté
" enseignante tout court " (2 février 2017, entrée en fonction en 1985) pour porter la casquette d'entraîneur en club. De ce fait, elle valorise le côté sportif, connoté plus masculin et atténue l'aspect scolaire, considéré comme davantage féminin. Ainsi, les élèves filles et garçons vivent des expériences différentes tout en apprenant à respecter les codes et normes propres à leur sexe. Les interactions verbales entre les enseignantes et leurs élèves participent à une socialisation scolaire différenciée et inégalitaire (Couchot-Schiex \& Trottin, 2005). D'ailleurs, quantitativement, plusieurs enseignantes reconnaissent interagir davantage avec les garçons.

De manière générale et d'après les dires des enseignantes, les élèves ne semblent pas dérangé.e.s par le genre de l'enseignante et ce dernier ne semble pas poser problème sauf dans certains cas spécifiques. Néanmoins, la plupart des enseignantes interrogées abordent la question de la drague amorcée par les élèves garçons, en racontant différentes anecdotes. Françoise relate une expérience récente, "la seule en 13 ans d'enseignement ", lors d'une leçon de rugby, sport qu'elle affectionne tout particulièrement : "Un élève a dit, quand j'ai voulu démontrer [...] : "mais vous ne pouvez pas, Madame”, [...], un élève respectueux pourtant, pas de problème de discipline; et puis il m'a dit "mais parce que vous êtes une femme” " (21 février 2017, entrée en fonction en 2004). Ainsi, l'enseignement d'une activité physique et sportive davantage «masculine » par une femme à des élèves garçons peut susciter de rares réactions d'opposition de la part de ces derniers.

De plus, les relations entre enseignantes d'éducation physique et élèves filles peuvent parfois générer de rares conflits, bien qu'en grande partie liés au jeune âge des enseignantes au moment des faits, d'après ces dernières. Le manque d'expérience dû à l'âge couplé au genre de l'enseignante et ce qu'il provoque est une thématique largement abordée par les enseignantes et étroitement liée à certaines problématiques issues de la perception des élèves, comme le raconte Céline qui, 
en prenant les classes de garçons pour des remplacements à ses débuts, sentait " [qu'] ils respectaient plus l'autorité d'un homme que d'une femme" (2 février 2017, entrée en fonction en 1985). De plus, la majorité des enseignantes ont abordé d'elles-mêmes la question du besoin de « faire ses preuves " face à leurs élèves, en l'occurrence aux garçons, comme Gaïa qui ressent «le besoin de prouver quelque chose, en tant que femme quand [elle] enseigne le sport ", c'est-à-dire qu'elle connaît son métier, qu'elle sait réfléchir, mais aussi qu'elle "sait faire une performance » (3 janvier 2017, entrée en fonction en 2012). Une performance attendue globalement davantage par les élèves garçons, comme le suggère Hanna. Eva estime que parfois, c'est bien parce que c'est une femme que les élèves la soumettent à des mises au défi ou compétitions. Nous pouvons ainsi constater que les élèves ont intégré l'hégémonie masculine dans le sport qu'ils/ elles reproduisent même à l'école, face à un individu hiérarchiquement supérieur. Ainsi, pour contrer ce type de conduite, la stratégie utilisée par ces enseignantes est de prouver qu'elles ont leur place dans le domaine sportif et qu'elles sont compétentes techniquement aussi.

\section{CONCLUSION}

L'absence de réglementation sur la mixité dans les systèmes scolaires suisses - ici dans le système genevois - semble permettre la réactualisation permanente de stéréotypes de genre. Selon nos analyses, ces derniers sont très opérants dans le fonctionnement de l'éducation physique de nos jours et président aux choix de certains établissements d'enseigner l'éducation physique sous une forme mixte ou non mixte. De fait, pour les enseignantes interrogées, l'enseignement de l'éducation physique est un métier accessible autant aux femmes qu'aux hommes, bien que les choses semblent plus complexes dès lors que l'on essaie de comprendre la puissance de certains stéréotypes et de certaines représentations genrées, touchant à la fois les enseignant·e·s et les activités enseignées elles-mêmes.

Si la profession enseignante est considérée comme égalitaire à Genève et si les femmes sont tout aussi bien reçues que les hommes et pareillement considérées pour enseigner l'éducation physique, lorsque ces enseignantes évoquent le fait d'être une femme dans le métier, c'est pour valoriser leurs atouts et leurs points forts - au risque d'utiliser parfois des stéréotypes dans leurs discours -, en comparaison à leurs homologues masculins. Cela démontre la volonté de ces femmes d'asseoir leur place dans le milieu, bien que cette dernière soit nécessaire et reconnue. En effet, ces enseignantes ne sont pas simplement des pâles copies de leurs collègues hommes, bien au contraire, elles cherchent à valoriser une autre manière d'enseigner, ce qui tend à alimenter les tendances différenciatrices du système.

Pour certaines d'entre elles, la non-mixité est une vraie solution pour assurer un enseignement de qualité, sans qu'il ne nous ait été possible de véritablement saisir si ce choix repose sur une véritable analyse des enjeux pédagogiques inhérents aux deux situations ou s'il s'agit davantage de la force d'une certaine habitude, voire de la réactualisation de stéréotypes. De plus, rappelons que c'est bien lorsque ces femmes enseignantes d'éducation physique sont confrontées aux élèves garçons que certaines d'entre elles, toutes générations confondues, se sentent mises à l'épreuve. Ainsi, nous avons constaté qu'être une femme enseignante d'éducation physique implique souvent une perception différenciée de cet enseignement et de la relation aux élèves, ce qui appelle à la réalisation d'autres travaux plus approfondis autour des différents systèmes scolaires helvétiques et pour comprendre les dynamiques de la féminisation d'une profession dans un contexte d'une société encore plutôt conservatrice en termes de droits des femmes.

Dans les faits, la situation genevoise, représentative des systèmes scolaires helvétiques qui n'ont pas adopté de réglementation stricte sur la mixité en éducation physique, renvoie aux 
conclusions de l'enquête mandatée par Michel Volondat dans le système scolaire français dans les années 1980, soit quelques années seulement après l'introduction de la mixité. En 1984, en France, « seuls $21 \%$ des enseignants n'ont que des classes mixtes, $42 \%$ ne travaillent jamais en mixité, $21 \%$ font des groupes de niveaux qui conduisent le plus souvent à un démixage " (Gleyse, 2010, p. 84). De fait, s'il existe des différences dans la perception du métier et si les usages et les mentalités se transforment lentement, il apparaît donc d'autant plus important que le cadre législatif et réglementaire soit clairement défini pour garantir un enseignement égalitaire aux élèves, ou alors que les autorités prennent des dispositions pour garantir la non-mixité dans l'ensemble des classes, en cherchant à imposer cette situation comme une nouvelle norme. Le maintien d'un flou certain autour de cette situation tend en effet à renforcer la logique intrinsèque du système sportif qui est bien celle d'une bicatégorisation claire et rigide.

\section{BibLIOGRAPHIE}

Bréau, A., Lentillon-Kaestner, V. \& Hauw, D. (2016). Le retour de la non-mixité à l'école. État des recherches, maintien des tabous et « doing gender ». Revue française de pédagogie, 194, 109-138.

Bréau, A. \& Lentillon-Kaestner, V. (2017). Les garçons face à la mixité et à la non-mixité en EPS. Revue eJRIEPS, 40, 4-30.

Burgener, L. (1984). L'éducation physique et les sports dans les lois fédérales. Revue militaire suisse, 129(11), 491-498.

Bussard, J.-C. (2001). Les manuels fédéraux et l'institutionnalisation de l'éducation physique. In C. Jaccoud \& T. Busset (dir.). Sports en forme, acteurs, contextes et dynamiques dinstitutionnalisation (pp. 51-62). Lausanne : Antipodes.

Bussard, J.-C. (2007). L'éducation physique suisse en quête d'identité (1800-1930). Paris : L'Harmattan.

Cacouault-Bitaud, M. (2007). Professeurs... mais femmes : carrières et vies privées des enseignantes du secondaire au $X X^{e}$ siècle. Paris : La Découverte.

Cattani, M. (2017). Quelque chose à prouver ? Etre une femme enseignante d'éducation physique au secondaire à
Genève, entre 1970 et aujourd'hui. Mémoire de master en sciences du sport, Université de Lausanne.

Cogérino, G. (dir.) (2005). Filles et garçons en EPS. Paris : Éditions Revue EPS.

Cogérino, G. \& Joux, M. (2005). Filles et garçons en EPS : regards d'hommes, regards de femmes. In G. Cogérino (dir.). Filles et garçons en EPS (pp. 221260). Paris : Éditions Revue EPS

Cogérino, G. (2007). Propos d'enseignants d'éducation physique face à la mixité. Staps, 75, 25-42.

Cordoba, A. \& Lenzen, B. (2018). L'institutionnalisation des pratiques corporelles en Suisse. Etude de l'assujettissement du corps dans les programmes scolaires. In M. Aceti, C. Jaccoud \& L. Tissot (dir.). Des corps en Suisse. Temps, lieux et gens (pp. 6-31). Neuchâtel : Alphil.

Couchot-Schiex, S. \& Trottin, B. (2005). Interactions enseignants/élèves : variations en fonction du sexe et du genre. In G. Cogérino (die.). Filles et garçons en EPS (pp. 163-179). Paris : Éditions Revue EPS.

Czáka, V. (2012). Le prof de gym peut-il être une femme ? Parcours d'enseignant·e.s romands, 18601920. In M. Aceti \& C. Jaccoud (dir.). Sportives dans leur genre? Permanences et variations des constructions genrées dans les engagements corporels et sportifs (pp. 113-126). Berne : Peter Lang.

Durand-Delvigne, A. \& Duru-Bellat, M. (1998). Mixité scolaire et construction du genre. In M. Maruani (dir.). Les nouvelles frontières de linégalité : Hommes et femmes sur le marché du travail (pp. 83-92). Paris : La Découverte.

Essen, M. V. \& Rogers, R. (2003). Écrire l'histoire des enseignantes. Enjeux et perspectives internationales. Histoire de l'éducation, 98, 5-36.

Fontaine, A. (2015). Aux heures suisses de l'école républicaine. Un siècle de transferts culturels et de déclinaisons pédagogiques dans l'espace franco-romand. Paris : Demopolis.

Gleyse, J. (2010). L'éducation physique comme analyseur de l'histoire de la mixité dans les écoles (18822008). Tréma, 32, 69-92.

Hofstetter, R., Schneuwly, B. \& Lussi Borer, V. (2009). Une formation professionnelle universitaire pour tous les enseignants. L'exemple de la Suisse au $\mathrm{XX}^{\mathrm{e}}$ siècle. Recherche et formation, 60, 25-38.

Lentillon, V. (2009). Les stéréotypes sexués relatifs à la pratique des activités physiques et sportives chez les adolescents français et leurs conséquences discriminatoires. Bulletin de psychologie, 62, 15-28.

Lentillon, V. \& Cogérino, G. (2005). Les inégalités entre les sexes dans l'évaluation en EPS : sentiment d'injustice chez les collégiens. STAPS, 68, 79-95. 
Lentillon-Kaestner, V., Deriaz D., Voisard, N. \& Allain, M. (2018). Noter en éducation physique ? Incidences sur l'enseignement et les élèves. Louvain-la-Neuve : EME Éditions.

Maruani, M. (1998). Les nouvelles frontières de linégalité : hommes et femmes sur le marché du travail. Paris : La Découverte.

Mennesson, C. (2005). Etre une femme dans le monde des hommes: socialisation sportive et construction du genre. Paris : L'Harmattan

Moreau, M.-P. (2011). Les enseignants et le genre : les inégalités hommes-femmes dans l'enseignement du second degré en France et en Angleterre. Paris : PUF.

Mosconi, N. \& Vouillot, F. (2013). Pourquoi la mixité fait-elle encore parler d'elle ? In M. Maruani (dir.). Travail et genre dans le monde : l'état des savoirs (pp. 71-79). Paris : La Découverte.

Quin, G. (2012). De la guerre et de l'éducation physique en Suisse à la fin des années 1930. Quelques jalons pour une histoire de l'essor de la formation des «Maîtres·ses spécialisé·e·s " pour l'éducation physique à Lausanne. In L. Robène (die.). Le sport et la guerre, XIX ${ }^{e}-X X^{e}$ siècles (pp. 379-387). Rennes : PUR.

Quin, G. (2015). Le tournant "sportif " de la gymnastique féminine helvétique (1960-1985). L'Association suisse de Gymnastique féminine entre spécialisation et professionnalisation. Revue suisse d'histoire, 65(3), 428-448.

Quin, G. (2016). L'odyssée du sport universitaire lausannois. Entre compétition et sport-santé. Paris : Glyphe.

Studer, B. (1996). «L'État c'est l'homme ». Politique, citoyenneté et genre dans le débat autour du suffrage féminin après 1945. Revue suisse d'histoire, 46(3), 356-382.

Szerdahelyi L. (2009). L'Éducation Physique et Sportive entre sport et mixité durant les années 68. Clio. Histoire, femmes et sociétés, 29, 119-129.

\section{ANNEXE. LISTE DES PARTICIPANTES À LA RECHERCHE}

\begin{tabular}{|c|c|c|c|}
\hline $\begin{array}{c}\text { Prénoms d'emprunt } \\
\text { des enseignantes }\end{array}$ & $\begin{array}{c}\text { Date de } \\
\text { naissance }\end{array}$ & $\begin{array}{c}\text { Début de la carrière } \\
\text { professionnelle }\end{array}$ & Vie professionnelle au moment de l'entretien \\
\hline Agnès & 1953 & 1975 & À la retraite \\
\hline Béatrice & 1960 & 1985 & Cycle d'orientation (secondaire I) \\
\hline Céline & 1963 & 1985 & École de culture générale (secondaire II) \\
\hline Daniela & 1973 & 2000 & Collège et école de commerce (secondaire II) \\
\hline Eva & 1978 & 2001 & École de culture générale (secondaire II) \\
\hline Françoise & 1978 & 2004 & Cycle d'orientation (secondaire I) \\
\hline Gaïa & 1986 & 2012 & Cycle d'orientation (secondaire I) \\
\hline Hanna & 1990 & 2014 & Cycle d'orientation (secondaire I) \\
\hline
\end{tabular}

ABSTRAcT: Made compulsory rather late, in 1972, physical education (PE) for girls in the Swiss school system is now an unquestioned reality across the whole of the country and throughout the time pupils are in compulsory education. However, school systems have never made any true reflection on the conditions of the inclusion of girls in this specific area of education. In this study, we offer an analysis of the given situation, attempting to understand the logics of female employment in PE teaching and the changes within a discipline, ones of whose distinctive features is that it conveys very powerful and still highly effective gender representations. To do this, we carried out a series of interviews with female teachers of different generations, starting in the 1970s, to map out the potential changes in the recruitment of female PE teachers in the Swiss canton of Geneva. Situated at the intersection of a history of $\mathrm{PE}$ and the sociology of a unique profession, our conclusions highlight the persistence of certain "gendered" representations, the slow transformation of a professional field, and the issues inherent to the preservation of the non-regulated status of co-education in PE and in the broader educational context.

KEYwORDs: physical education, female teachers, Switzerland, gender 


\section{Zusammenfassung: Als Sportlehrerin im Kontext ungeregelter Gemischtgeschlechtlichkeit: eine explorative Studie in der Schweiz (Genf)}

Der 1972 erst spät verpflichtend gewordene Sportunterricht für Mädchen in den Schulsystemen der Schweiz ist inzwischen eine Bereicherung und Realität im gesamten Staatsgebiet und für die gesamte Dauer der Schulpflicht. Es wurde jedoch nie über die Bedingungen dieses Eintritts der Mädchen in so ein besonderes Schulfach nachgedacht. In diesem Kontext wollen wir deshalb die Logik der Einbindung von Frauen in den Sportunterricht und die Veränderungen in einem Schulfach, zu dessen Besonderheiten es gehört, sehr starke und noch sehr wirkungsvolle Geschlechterrepräsentationen zu vermitteln, analysieren. In diesem Zusammenhang haben wir Interviews mit Lehrerinnen verschiedener Generationen seit den 70er Jahren geführt, um die möglichen Veränderungen des Engagements von Sportlehrerinnen für den Kanton Genf zu ermitteln. Basierend auf der Verbindung einer Geschichte des Sportunterrichts mit einer Soziologie eines einzelnen Berufsstandes unterstreichen unsere Schlussfolgerungen zugleich den Fortbestand bestimmter geschlechtsspezifischer Repräsentationen, die langsamen Veränderungen eines Berufs und die mit der Aufrechterhaltung einer Nichtregulierung der Gemischtgeschlechtlichkeit im Sportunterricht und im allgemeinen schulischen Kontext verbundenen Schwierigkeiten.

SCHLAGWÖRTER: Sportunterricht, Lehrerinnen, Schweiz, Gender

\section{Resumen: Ser profesor de educación física en un contexto no regulado de integración : estudio exploratorio en Suiza (Ginebra)}

La enseñanza de la educación física para niñas en los sistemas escolares de Suiza, es obligatoria a partir de 1972, actualment es una realidad en todo el territorio nacional y durante toda la escolarización como una clase obligatoria. Sin embargo, los sistemas escolares suizos nunca han reflexionado sobre las condiciones del ingreso de las niñas en esta disciplina en la escuela. En este contexto, nuestra ambición es proponer un análisis de esta situación, investigando comprender la lógica de la participación de las mujeres en la enseñanza de la educación física y los cambios de esta disciplina a nivel escolar, que incluyen la transmición de representaciones de género que son muy poderosas y aún muy efectivas. En este contexto, nos comprometimos a realizar entrevistas con docentes de diferentes generaciones a partir de los años de 1970 para investigar e identificar las posibles transformaciones del compromiso de las profesoras de educación física del Cantón de Ginebra. Al comparr la historia de la educación física y la sociología de esta profesión muy singular, nuestras conclusiones enfatizan la permanencia de ciertas representaciones "de género", las lentas transformaciones de esta profesión y las dificultades inherentes a mantenere una no regulación de la situación mixta en educación física y ampliamente en el contexto escolar.

Palabras Claves: Educación física, profesoras mujeres, Suiza, género

\section{Riassunto: Essere insegnante di educazione fisica in un contesto non regolamentato di co-educazione: studio esplorativo in Svizzera (Ginevra)}

Resa obbligatoria tardivamente, nl 1972, l'educazione fisica per le ragazze nei sistemi scolastici della Svizzera è ormai un'acquisizione e una realtà sull'insieme del territorio nazionale e per tutta la durata della scolarità obbligatoria. Tuttavia, i sistemi scolastici elvetici non sono mai stati sottoposto ad una riflessione sulle condizioni di questa entrata delle ragazze in una branca singolare della Scuola. In questo quadro, la nostra ambizione è di proporre un'analisi di questa situazione, cercando di comprendere le logiche dell'impegno delle donne nell'insegnamento dell'educazione fisica e le mutazioni di una branca scolastica, di cui una delle specificità è di veicolare delle rappresentazioni di genere molto potenti e ancora molto effettive. In questo quadro, noi abbiamo intrapreso di realizzare delle interviste con insegnanti di differenti generazioni a partire dagli anni 1970 per cercare di circoscrivere le potenziali trasformazioni dell'impegno delle insegnanti di educazione fisica per il cantone di Ginevra. All'incrocio di una storia dell'educazione fisica e di una 
sociologia di una professione singolare, noi concludiamo sottolineando nello stesso tempo la permanenza di certe rappresentazioni di «genere», le lente trasformazioni di un mestiere e le difficoltà inerenti al mantenimento di una non-regolazione della situazione di co-educazione in educazione fisica e più largamente in cotesto scolastico.

Parole Chiave: donne insegnanti, educazione fisica, genere, Svizzera 